\title{
Advanced parallel and distributed computing for big urban
} data

The Journal of Supercomputing gratefully acknowledges the editorial work of the scholars listed below on the special issue entitled "Advanced Parallel and Distributed Computing for Big Urban Data".

\section{Corresponding Guest Editor}

\section{Xiaohui Yuan}

University of North Texas, USA

Email: xiaohui.yuan@unt.edu

\section{Guest Editors}

\section{Baofu Fang}

Hefei University of Technology, China

Email: fangbf@hfut.edu.cn

\section{Mohamed Elhoseny}

Mansoura University, Egypt

Email: mohamed_elhoseny@mans.edu.e.g. 